\title{
Molecular mechanisms of the analgesic action of Wu-tou Decoction on neuropathic pain in mice revealed using microarray and network analysis
}

\author{
Yan-qiong ZHANG\#, * , Chao WANG\#, Qiu-yan GUO, Chun-yan ZHU, Chen YAN, Dan-ni SUN, Qiong-hong XU, Na LIN* \\ Institute of Chinese Materia Medica, China Academy of Chinese Medical Sciences, Beijing 100700, China
}

\begin{abstract}
Wu-tou Decoction (WTD) is a classic herbal formula in traditional Chinese medicine for the treatment of joint diseases, neuropathic pain (NP) and inflammatory pain. In this study we investigated whether WTD produced analgesic action in a mouse spinal nerve ligation (SNL) model and elucidated the underlying molecular mechanisms. Mice were subjected to SNL and orally treated with WTD $\left(3.15,6.30\right.$ or $\left.12.60 \mathrm{~g} \cdot \mathrm{kg}^{-1} \cdot \mathrm{d}^{-1}\right)$ for $21 \mathrm{~d}$. SNL induced mechanical hyperalgesia and heat hyperalgesia characterized by rapid and persistent pain hypersensitivity. In addition, the expression levels of IL-1 $\beta$, TNF- $\alpha$, CCL2 and CXCL1 in the spinal cord dorsal horn were dramatically increased on the $10^{\text {th }} \mathrm{d}$ post-surgery. Oral administration of WTD dose-dependently suppressed both mechanical and heat hyperalgesia as well as the expression levels of inflammatory cytokines in the spinal cord dorsal horn on the $21^{\text {st }} \mathrm{d}$ post-surgery. Then whole-genome microarray analyses were conducted to detect the gene expression profiles of spinal cord dorsal horn in SNL mice with or without WTD treatment. After construction of the WTD-SNL-network and topological analysis, a list of candidate target genes of WTD acting on SNL-induced NP was identified and found to be functionally enriched in several glial cell activation-related pathways and neuroinflammatory pathways. Our data have clarified the gene expression patterns in the mouse spinal cord under the NP condition. We also demonstrate the analgesic action of WTD through suppression of glial cell activation and neuroinflammation, which suggest the potential of WTD as a promising candidate for the treatment of NP.
\end{abstract}

Keywords: Wu-tou Decoction; herbal formula; neuropathic pain; spinal nerve ligation; hyperalgesia; inflammatory cytokines; microarray analysis; network pharmacology; glial cell activation; neuroinflammation

Acta Pharmacologica Sinica (2018) 39: 988-997; doi: 10.1038/aps.2017.110; published online 17 Aug 2017

\section{Introduction}

Neuropathic pain (NP) is defined as pain caused by injuries or diseases of the peripheral and central nervous system, according to the International Association for the Study of Pain ${ }^{[1]}$. Estimation of the incidence and prevalence of NP is difficult, owing to the lack of simple diagnostic criteria. Although a growing number of studies identify several candidate targets for NP treatment, including ion channels, G-protein-coupled receptors, and kinases in neurons and glial cells localized along the nociceptive pathways ${ }^{[2,3]}$, NP is the most difficult type of chronic pain to manage in the pain clinic ${ }^{[4,5]}$ because the transcriptional or translational regulatory mechanisms underlying the expression and functional changes of these

\footnotetext{
\# These authors contributed equally to this work.

* To whom correspondence should be addressed.

E-mail nlin@icmm.ac.cn (Na LIN); yqzhang@icmm.ac.cn (Yan-qiong ZHANG)

Received 2017-04-03 Accepted 2017-05-19
}

candidate targets have not been fully elucidated.

Research on whole-genome transcription profiling obtained by microarray technology can determine RNA expression changes in tissues under different conditions and be used to select candidate molecules at the genome wide level. Such approaches have been extensively used to provide insights into the genetic mechanisms underlying the pathogenesis of NP. For example, Rojewska et $a l^{[6]}$ have confirmed changes in gene expression, identified by a microarray analysis, in the spinal cord and selected seven genes as candidate NP-related genes. Vallejo et al ${ }^{[7]}$ have used microarray analysis to identify a list of highly significant interconnected genes and pathways responsive to injury and/or an electric field in the spinal cord and dorsal root ganglion. Jiang et al ${ }^{[8]}$ have performed lncRNA microarray and mRNA microarray analyses and have uncovered the expression patterns of lncRNAs and mRNAs in the mouse spinal cord under the NP condition. These findings indicate the efficiency of microarray technology for the determination of the molecular mechanisms associated with the 
development and progression of NP as well as for the discovery of novel therapeutic targets in this disease.

Wu-tou Decoction (WTD), which consists of Radix Aconiti, Herba Ephedrae, Radix Astragali, Raidix Paeoniae Alba and Radix Glycythizae, is a classic herbal formula for the treatment of joint diseases, NP and inflammatory pain ${ }^{[9-12]}$. Our previous studies have confirmed the disease-modifying anti-rheumatic effects of WTD on rheumatoid arthritis (RA) through regulation of the imbalance in the nervous, endocrine and immune systems ${ }^{[13,14]}$ and have identified the pathway of PPAR-gamma coactivators in thermogenesis as one of the potential pharmacological targets of WTD for the individualized treatment of $\mathrm{RA}^{[15]}$. We have also revealed the analgesic effects of WTD in chronic inflammatory pain, which manifest partially through inhibition of the activities of TRPV1, TRPA1, and TRPM8 ${ }^{[10]}$. However, there is still little NP-related experimental evidence regarding the analgesic effects of WTD.

Chinese herbal formulae, characterized by multiple components, multiple targets and holistic regulations, have been widely utilized in clinics for centuries. However, clarification of the complex compositions and underlying molecular mechanisms of herbal formulae through traditional experimental methods based on the "one gene, one drug, one disease" paradigm is difficult. Accumulating studies indicate that Chinese herbal formulae achieve their particular therapeutic efficacy by modifying the imbalanced disease-associated network through the interactions of their active components and corresponding targets. Therefore, our research group has proposed a workflow that involves the integration of traditional Chinese medicine and network pharmacology, highlighting a "network target, multi-component therapeutics" paradigm to effectively reveal the modes of action of different Chinese herbal formulae ${ }^{[13-21]}$.

In the current study, we sought to investigate whether WTD exhibits analgesic effects in a spinal nerve ligation (SNL) model in mice and to reveal the possible underlying molecular mechanisms. Figure 1 illustrates a schematic diagram of our systematic strategies: first, the SNL mouse model was constructed, and WTD was orally administered at different dosages by syringe feeding; second, the analgesic effects of WTD on SNL mice at different time points were evaluated by a behavioral assessment and Enzyme-Linked Immunosorbent Assay (ELISA); third, tissues from the spinal cord dorsal horn in SNL mice were collected and subjected to whole-genome microarray analyses, followed by selection of the optimal dosage and time point for WTD administration; fourth, SNLinduced NP-related genes and WTD-treatment dysregulated genes were identified by screening differentially expressed genes (DEGs) among different groups by using the $\mathrm{R}$ software package; and fifth, the WTD-SNL-network was constructed, and the candidate target genes of WTD that act on NP were identified according to a topological comparison, functional enrichment analysis and literature search.

\section{Materials and methods} Ethics statement

The experimental protocols in the current study were approved by the Research Ethics Committee of the Institute of Chinese Materia Medica, China Academy of Chinese Medical Sciences, Beijing, China. All animal studies were carried out in accordance with the guidelines and regulations for the care and use of laboratory animals of the Center for Laboratory Animal Care, China Academy of Chinese Medical Sciences. Every effort was made to minimize the number of animals used and any pain or discomfort experienced by the animals.

\section{Animals and treatment}

Male ICR mice ( 8 weeks old) were obtained from the Experimental Animal Center of Beijing University, Beijing, China (License No SCXK-2012-0004). The mice were housed under standard conditions at an ambient temperature of $22 \pm 1{ }^{\circ} \mathrm{C}$ with $60 \%$ humidity under an automatically controlled $12 \mathrm{~h}$ light/ dark cycle and were given a regular laboratory rodent diet and water ad libitum. Following acclimatization for one week, the mice were anesthetized with sodium pentobarbital (40-50 $\mathrm{mg} / \mathrm{kg}$, ip) and the SNL models were constructed according to a previous description ${ }^{[22]}$. Briefly, the L4 and L5 spinal nerves were exposed after the removal of a portion of the left L6 transverse process. In addition, the L5 spinal nerve was carefully isolated without injury to the L4 spinal nerve and tightly ligated with a 6-0 silk thread. After the pain-related surgeries, mice were divided into five groups (10 per group): Sham, SNL group, SNL-WTD (3.15 g/ kg) group, SNL-WTD (6.30 g/ kg) group, and SNL-WTD $(12.60 \mathrm{~g} / \mathrm{kg})$. Among these, mice in the Sham group were subjected to similar surgical procedures, but no L5 SNL was carried out. The water extract of WTD, of which the chemical components were identified in our previous study ${ }^{[10]}$, was prepared for the treatment of the SNL mice. Treatment WTD was performed for $21 \mathrm{~d}$ via oral administration starting on the first day that the animals underwent surgery. Dosage selection for low-, middle- and high-WTD were nearly equivalent to $0.5,1$ and 2 times the daily dosage, which were $3.15 \mathrm{~g} / \mathrm{kg}, 6.30 \mathrm{~g} / \mathrm{kg}$ and $12.60 \mathrm{~g} / \mathrm{kg}$, respectively. In the Sham and SNL groups, WTD was replaced by an equal volume of distilled water. In each group, 6 mice were used for the behavioral assessment and 4 mice were used for ELISA.

\section{Measurement of mechanical hyperalgesia}

Mechanical hyperalgesia of mice in different groups was measured according to our previous study ${ }^{[10]}$. The $50 \%$ paw withdrawal threshold was analyzed at 1 (1 h), $3(0,1,2,3,4$, and $24 \mathrm{~h}), 7,10,14,17$ and $21 \mathrm{~d}$ after WTD administration.

\section{Measurement of heat hyperalgesia}

Heat hyperalgesia of mice in different groups was measured according to our previous study ${ }^{[10]}$. The paw withdrawal latency was measured at 2 (1 h), 4 (0, 1, 2, 3, 4, and $24 \mathrm{~h}), 8,12$, 16,18 , and $20 \mathrm{~d}$ after WTD administration. The interval of the paw withdrawal latency measurement was $5 \mathrm{~min}$.

\section{ELISA}

ELISA was performed to measure the interleukin (IL)- $1 \beta$, tumor necrosis factor alpha (TNF-a), chemokine (C-C motif) 


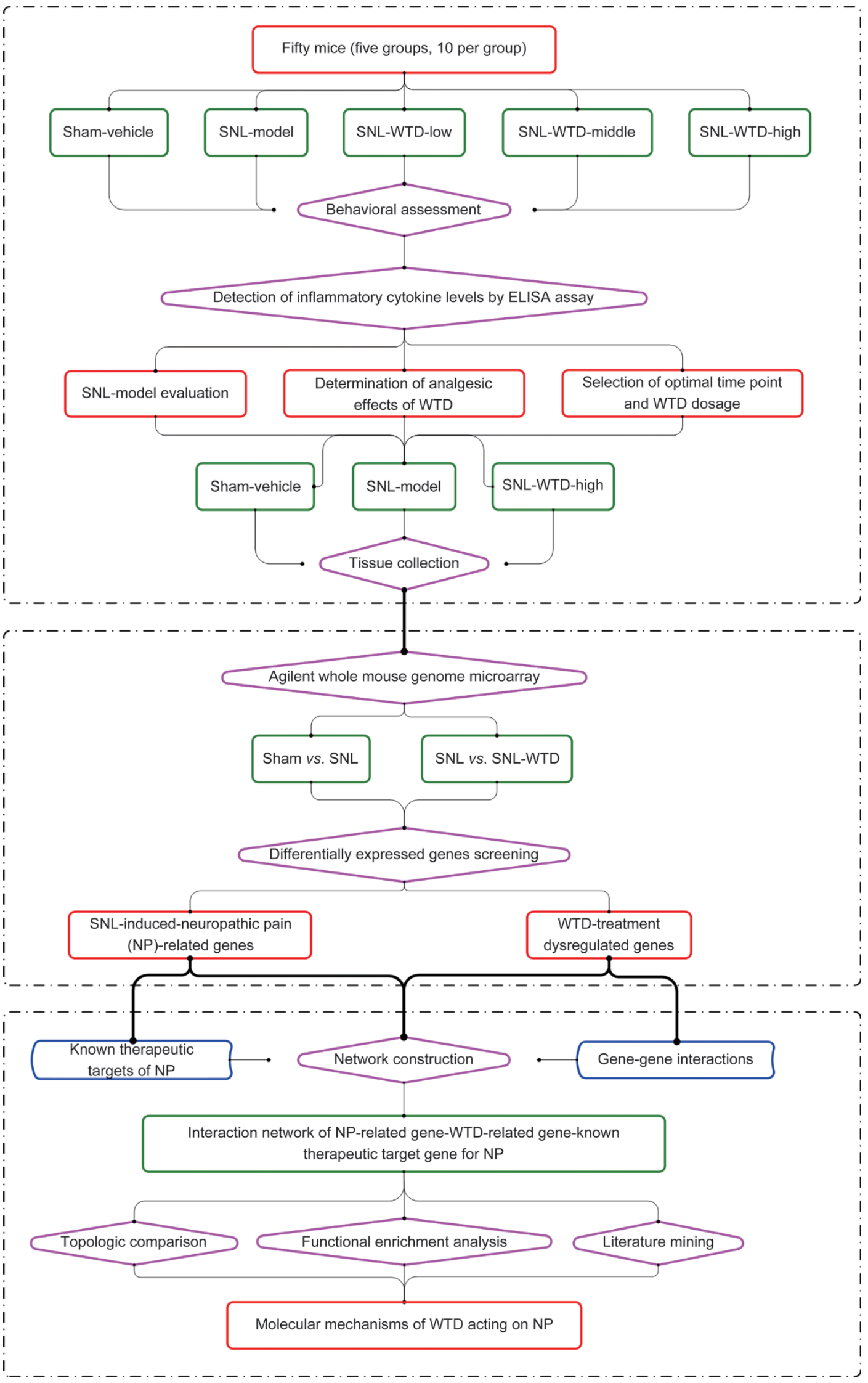

Figure 1. A schematic diagram of the systematic strategies for the determination of the pharmacological mechanisms of the herbal formula Wu-tou Decoction on peripheral neuropathic pain. 
ligand 2 (CCL2) and chemokine (C-X-C motif) ligand 1 (CXCL1) levels in the isolated L5 dorsal horn from SNL mice by using ELISA kits purchased from Elabscience Biotechnology Co, Ltd (Wuhan, China). All ELISAs were performed according to the manufacturer's protocols and measured at $450 \mathrm{~nm}$.

\section{Whole-genome microarray analysis}

Spinal cord dorsal horn tissues were collected the mice in the Sham, SNL and SNL-WTD (12.60 g/kg) groups; immersed in TRIzol (Invitrogen, CA, USA); and immediately frozen in liquid nitrogen for whole-genome microarray detection. Total RNA was extracted and purified using an RNeasy micro kit (Cat\#74004, QIAGEN, GmBH, Germany) according to the manufacturer's instructions, and the RNA Integrity Number (RIN) was determined to assess RNA integration by an Agilent Bioanalyzer 2100 (Agilent Technologies, Santa Clara, CA, USA). The RIN is defined to help scientists estimate the integrity of total RNA samples. The RNA concentration and purity were assayed by absorbance values at 260 and $280 \mathrm{~nm}$ by using a NanoDrop ND-2000 Spectrophotometer (Thermo Fisher Scientific, Inc, Wilmington, DE, USA) and Agilent Bioanalyzer 2100 (Agilent Technologies, Santa Clara, CA, USA). RNA integrity was verified by electrophoresis on $2 \%(\mathrm{~m} / \mathrm{v})$ agarose gels.

Agilent Whole Mouse Genome Microarray $4 \times 44 \mathrm{~K}$ gene chips, including 41174 coding gene probes, were used in the experiments. The total RNA of different groups was individually hybridized with gene chips. Briefly, each chip was hybridized with $1.65 \mu \mathrm{g}$ of Cy3-labeled cRNA by using a Gene Expression Hybridization Kit (Cat\# 5188-5242, Agilent Technologies, Santa Clara, CA, USA) in a Hybridization Oven (Cat\# G2545A, Agilent Technologies, Santa Clara, CA, USA) according to the manufacturer's instructions. After 17 $\mathrm{h}$ of hybridization, the chips were washed in staining dishes (Cat\# 121, Thermo Shandon, Waltham, MA, USA) with a Gene Expression Wash Buffer Kit (Cat\# 5188-5327, Agilent Technologies, Santa Clara, CA, USA) following the manufacturer's instructions. The chips were scanned with an Agilent Microarray Scanner (Cat\# G2565CA, Agilent Technologies, Santa Clara, CA, USA) with the default settings, Dye channel: Green, Scan resolution $=5 \mu \mathrm{m}$, PMT 100\%, 10\%, 16 bit. The data were extracted with Feature Extraction software 10.7 (Agilent Technologies, Santa Clara, CA, USA). Raw data were normalized by the Quantile algorithm, GeneSpring Software 12.6.1 (Agilent Technologies, Santa Clara, CA, USA). Microarray hybridization was carried out by Shanghai Biotechnology Corporation, Shanghai, China. Gene expression microarray data of GSE97076 were obtained from the National Center of Biotechnology Information (NCBI) Gene Expression Omnibus (http://www.ncbi.nlm.nih.gov/geo/query/acc. cgi?acc=GSE97076).

\section{DEG screening}

Significant DEGs of the sham vs SNL groups and SNL vs SNLWTD $(12.60 \mathrm{~g} / \mathrm{kg})$ groups were identified using the criteria of a $\mid \log 2$-fold change $(\mathrm{FC}) \mid>0.5$ and $P$ value $<0.05$. Hierarchical clustering analysis was performed to screen DEGs using the heat map package in $\mathrm{R}$ (version 1.0.2, R Core Team, Vienna, Austria). Cluster analysis of the DEGs was performed by Cluster 3.0 based on the Euclidean distance.

\section{Collection of known NP-related genes}

Known NP-related genes were obtained from the following two databases: (1) the DrugBank database ${ }^{[23]}$ (http://www. drugbank.ca/, version 5.0). We used only the drug-target interactions whose drugs are FDA approved for the treatment of NP and whose targets are human genes/proteins. In total, we obtained 385 known therapeutic targets of NP. (2) The Online Mendelian Inheritance in Man (OMIM) database ${ }^{[24]}$ (http:/ / www.omim.org/, last updated: October 31, 2014). We searched the OMIM database with the keyword "neuropathic pain" and found 540 known NP-related targets. After deleting redundancies, 880 known NP-related genes remained and were used in this study. The list of known NP-related genes is provided in Supplementary Table S1.

\section{Network construction and analysis}

Gene-gene interaction networks were constructed on the basis of the public database STRING (Search Tool for Known and Predicted Protein-Protein Interactions, version 10.0, http:/ / string-db.org/). Gene-gene interactions with a combined score higher than the median value of all of the combined scores were selected in this study. The interaction networks were visualized by Navigator software (version 2.2.1). A node was defined as a gene in the network; edge was defined as the interaction between two genes; and hub was defined as a node of topological importance. Four topological features, the node degree, betweenness, closeness and k-coreness, were calculated to evaluate the topological importance of a node in the interaction networks. The definitions of the four network topological features are provided in Supplementary File S1-section1.

\section{Function and pathway enrichment analyses}

Kyoto Encyclopedia of Genes and Genomes (KEGG) pathway enrichment analyses were performed based on the Database for Annotation, Visualization and Integrated Discovery52 (DAVID, http://david.abcc.ncifcrf.gov/home.jsp, version 6.7) and pathway data obtained from the FTP service of KEGG (http://www.genome.jp/kegg/, last updated: Oct 16, 2012). Only KEGG pathways with $P$-values < 0.05 (corrected using the Bonferroni method) were selected.

\section{Statistical analysis}

Statistical analysis were performed using SPSS statistical software (Version 13.0, SPSS, Inc, Chicago, IL, USA). All experiments were performed in triplicate. The data are represented as the mean \pm SEM. Differences among groups in the behavioral assessment were statistically analyzed by twoway ANOVA followed by Bonferroni post-tests. Differences among the groups in ELISA were assessed by $t$ tests. $P$-values 
less than 0.05 were considered significant.

\section{Results}

\section{Identification of the NP model}

As shown in Figures 2A and 2B, SNL induced rapid (1-2 d) and persistent (>21 d) mechanical hyperalgesia and heat hyperalgesia in the SNL group compared with the sham group (all $P<0.05$ ). In addition, we also detected the expression levels of inflammatory cytokines secreted by microglia (IL-1 $\beta$ and TNF- $\alpha$ ) and astrocytes (CCL2 and CXCL1) in the spinal cord dorsal horn at $10 \mathrm{~d}$ (maintenance phase) after SNL. The data shown in Figure 3 demonstrated significantly higher expression of IL-1 $\beta$, TNF- $\alpha$, CCL2 and CXCL1 proteins in SNL mice than in sham-treated mice (all $P<0.05$ ), thus indicating that glial activation was induced in the spinal cord by SNL.

\section{WTD dose-dependently attenuates SNL-induced NP}

To determine whether WTD attenuates SNL-induced NP, different doses $(3.15,6.30$ and $12.60 \mathrm{~g} / \mathrm{kg})$ of WTD were orally administered once per day for $21 \mathrm{~d}$, and the behavioral assess- ment was performed $1 \mathrm{~h}$ after each drug treatment. As shown in Figure 2A, WTD (3.15-12.60 g/ kg) dose-dependently attenuated mechanical allodynia, which lasted for at least $2 \mathrm{~h}$, with an optimal inhibitory effect $1 \mathrm{~h}$ post-WTD administration. This anti-allodynia effect was maintained for at least 7 $\mathrm{d}$ when the drug was administered daily. Importantly, WTD decreased mechanical allodynia with a time-course effect profile similar to that of the first day, thus indicating the absence of drug tolerance. Although WTD $(12.60 \mathrm{~g} / \mathrm{kg})$ significantly attenuated mechanical allodynia in SNL-induced hypersensitivity, it did not alter the baseline thresholds in sham control mice, thus suggesting that WTD has a specific role under NP conditions. In addition, we also investigated the anti-hyperalgesia effects of WTD in a radiant heat plantar test. Figure 2B shows the dose-dependent inhibition of heat hyperalgesia by WTD (3.15-12.60 g/ kg) administration, with a similar time course-effect compared with that of mechanical allodynia. Moreover, WTD $(12.60 \mathrm{~g} / \mathrm{kg})$ produced no analgesic effect in the sham control mice in the radiant heat test, thus further ruling out the possibility of drug tolerance and identifying

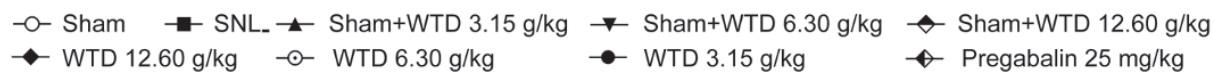

A

$\#$

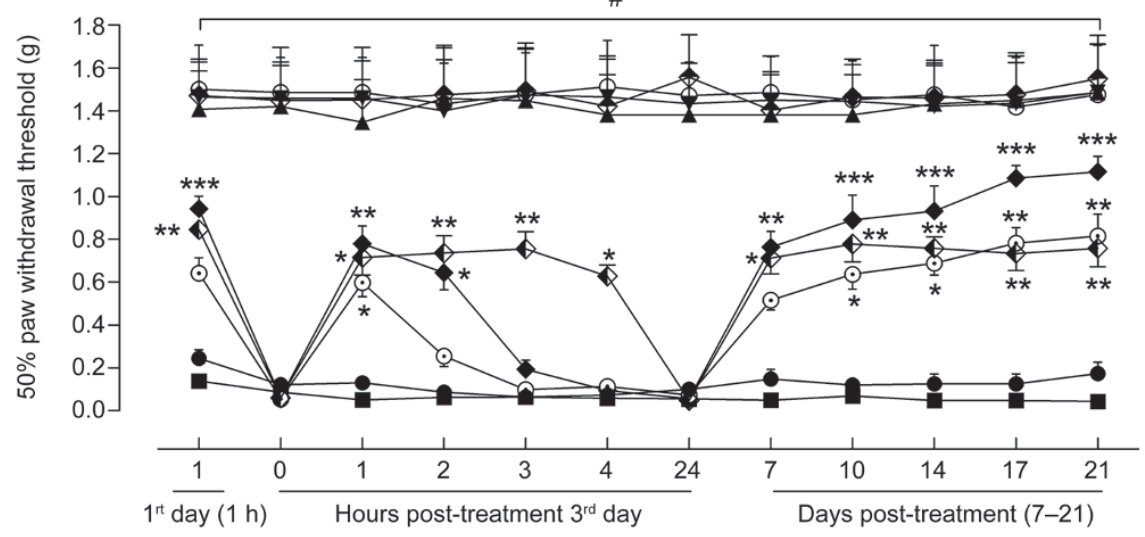

B

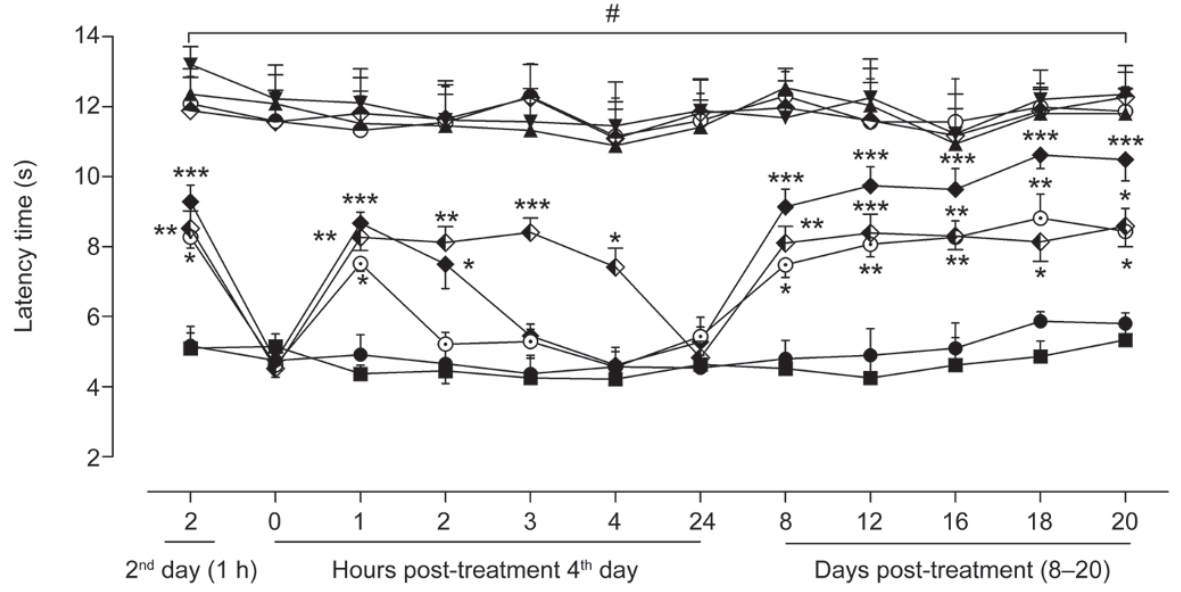

Figure 2. Behavioral assessment in different groups. (A) Changes in mechanical hypersensitivity among the Sham, SNL, sham-WTD-treatment and SNLWTD-treatment groups. (B) Changes in heat hypersensitivity among the sham, SNL, sham-WTD-treatment and SNL-WTD-treatment groups. The data are represented as the mean \pm SEM $(n=6)$. ${ }^{\#} P<0.05$ versus the sham group; ${ }^{*} P<0.05,{ }^{* *} P<0.01$, and ${ }^{* * *} P<0.001$ versus the SNL group, respectively. 
A

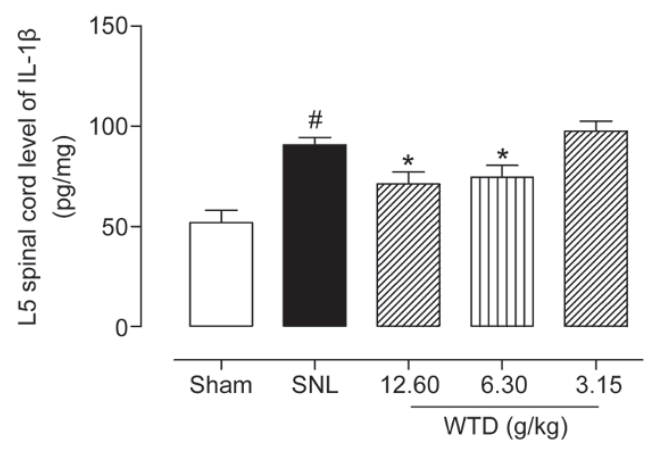

C

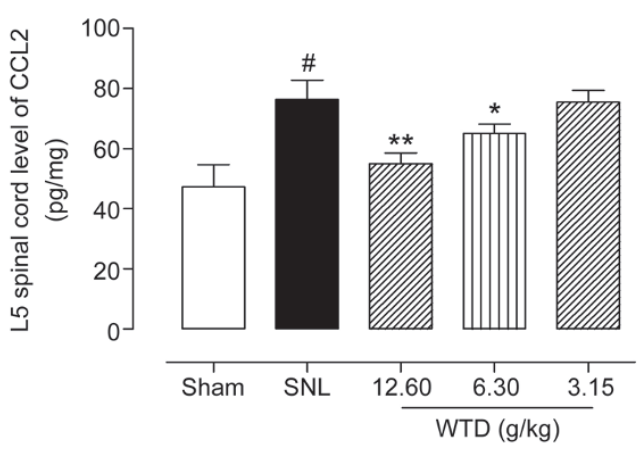

B
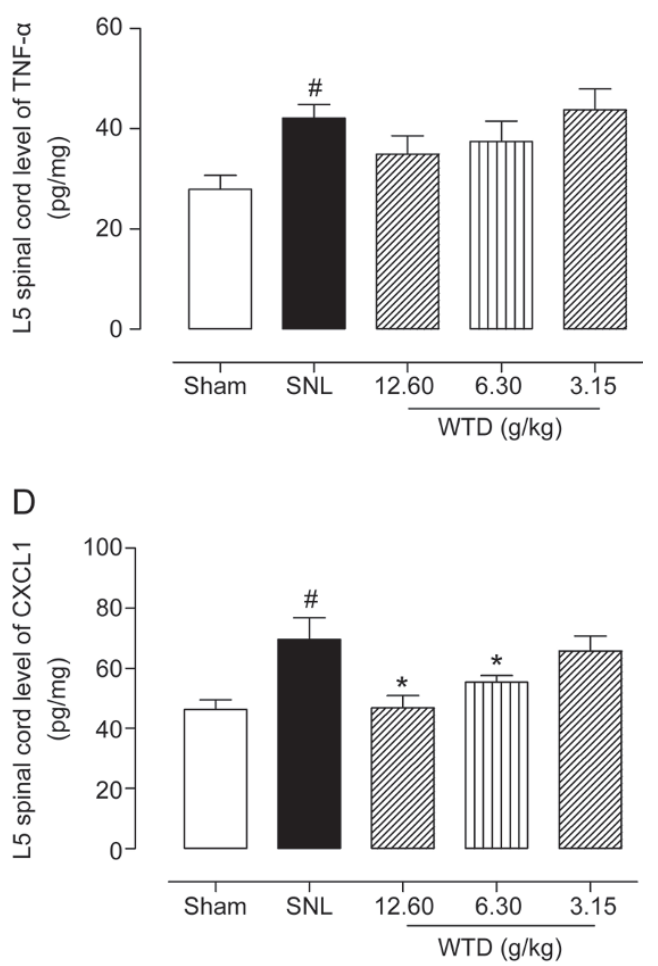

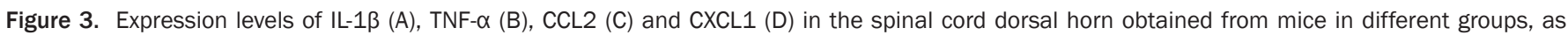

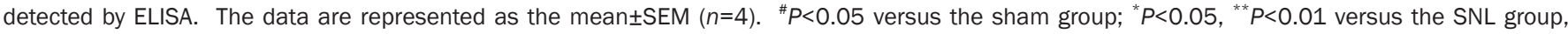
respectively.

its unique role in neuropathic pain normalization (Figure 2). Furthermore, WTD treatment clearly decreased the expression levels of IL-1 $\beta$, CCL2 and CXCL1 in the spinal cord dorsal horn in SNL mice dose-dependently (all $P<0.05$, Figure 3).

NP-related genes are implicated in glial cell activation, neuroimmune responses and neuroinflammation

A total of 567 DEGs (331 upregulated and 236 downregulated) were identified in SNL and sham-operated mice (Supplementary Table S2). Among these, 18 genes (ADCY1, ADRA2A, B4GALT3, BRAF, BTG2, CHRNA4, DYNC1H1, EGFR, GLO1, HTR1D, IL1R1, PDGFRA, PDPK1, PGR, PNPLA6, SCN1B, TEK, WARS) were identified as known NP-related genes. In addition, unsupervised hierarchical clustering (Figure 4A) and scatter plot (Figure 4B) analyses of all dysregulated genes showed good differentiation of SNL and sham-treatment samples.

Then, we constructed the SNL-induced NP imbalance network by using the interaction information from SNL differentially expressed genes and known NP-related genes. This network consists of 756 nodes and 4774 edges. According to the degree values of the nodes in the network ${ }^{[13-15]}, 248$ hub genes were selected as SNL-induced NP-related genes (Supplementary Table S3), which contained many known genes involved in the pathways related to glial cell activation (Neuroactive ligand-receptor interaction, Calcium signaling pathway, Neurotrophin signaling pathway, Gap junction and
Long-term potentiation), neuro-immune responses (Fc epsilon RI signaling pathway and $\mathrm{T}$ cell receptor signaling pathway) and neuroinflammation (MAPK signaling pathway, Cytokinecytokine receptor interaction, Jak-STAT signaling pathway, Chemokine signaling pathway, NOD-like receptor signaling pathway and Toll-like receptor signaling pathway) according to the pathway enrichment analysis (all $P<0.05$, Figure $4 C$ ).

WTD exerts an analgesic effect on SNL-induced NP through suppression of glial cell activation and neuroinflammation

A total of 442 DEGs (171 upregulated and 271 downregulated) were identified from the comparison between WTD-treatment and SNL mice (Supplementary Table S4). In addition, unsupervised hierarchical clustering (Figure 5A) and scatter plot (Figure 5B) analyses of all dysregulated genes showed good differentiation of WTD-treatment and SNL samples.

Next, the WTD-SNL-network was constructed on the basis of the links among the WTD-treatment dysregulated genes and SNL-induced-NP-related genes. This network consists of 375 nodes and 3077 edges. After the calculation of the 4 topological features in the network (degree, closeness, betweenness and k-coreness), 94 major nodes were identified because their 'Degree', 'Betweenness', 'Closeness' and 'K coreness' were all larger than the corresponding median values (Supplementary Table S5). Pathway enrichment analysis based on the KEGG pathway database showed that the major nodes in the WTDSNL-network were significantly associated with the path- 
A

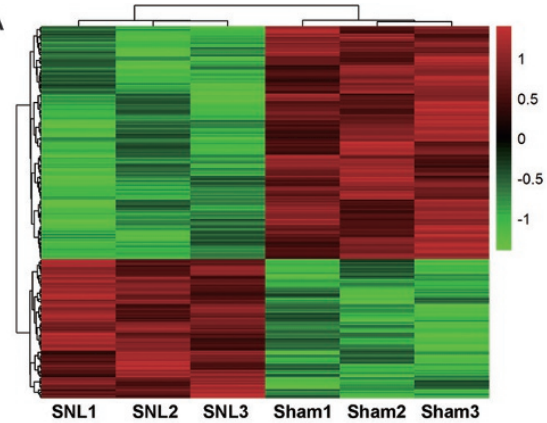

$\mathrm{B}$

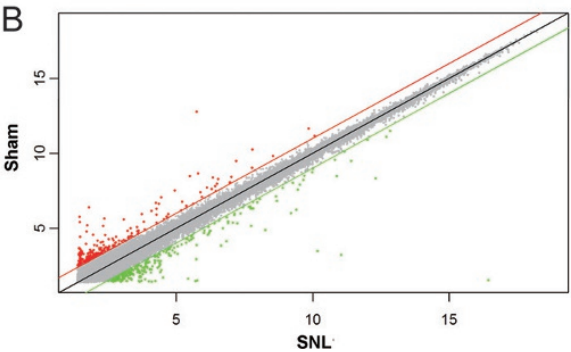

C Physiological state $\longrightarrow$ Neuropathic pain state
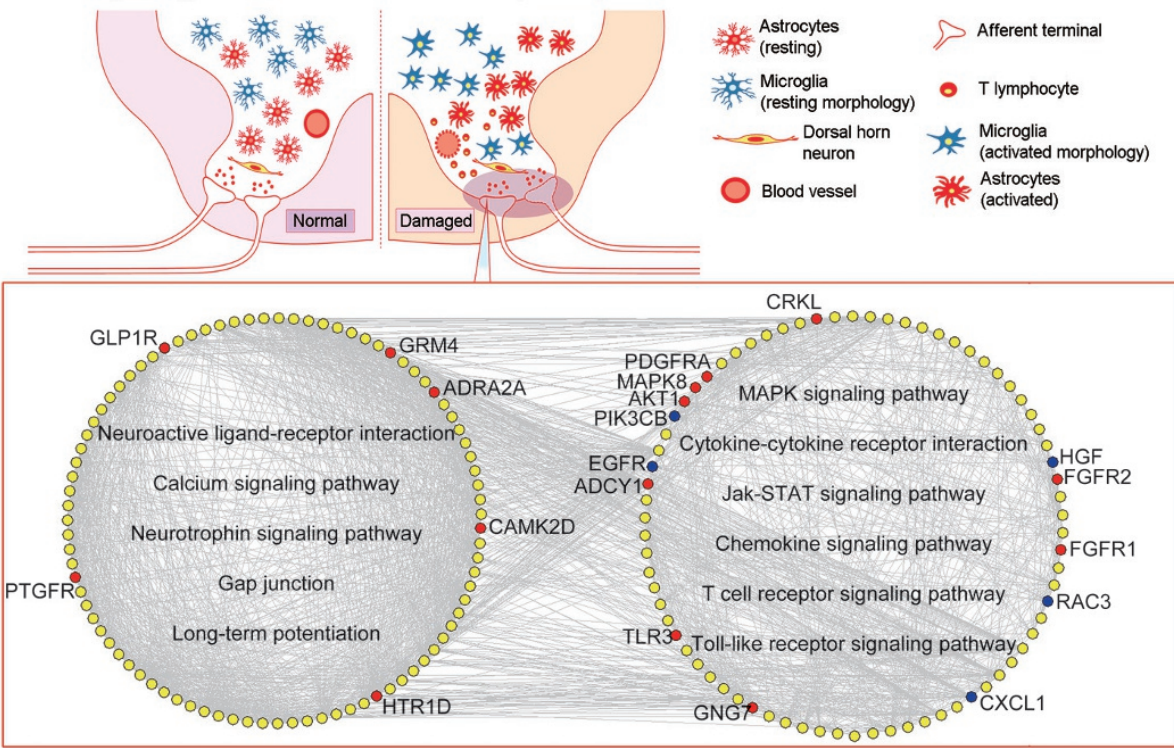

Figure 4. NP-related genes are implicated in glial cell activation, neuro-immune responses and neuroinflammation. (A) A heat map showing the hierarchical clustering of mRNAs, whose expression changes were changed by more than two-fold in the comparison between the sham group and the SNL group. In the clustering analysis, up- and downregulated genes are colored in red and green, respectively. (B) A scatter plot comparing the global mRNA expression profile in the spinal cord between SNL and sham mice. Red and green lines, respectively, indicate two-fold differences in upand downregulated genes. (C) The SNL-induced NP imbalance network constructed on the basis of interaction information among SNL differentially expressed genes and known NP-related genes. All nodes were hubs of the SNL-induced NP imbalance network. Red and blue nodes, respectively, refer to the upregulated and downregulated genes in the SNL group compared with the sham group. Yellow nodes refer to the known NP-related genes.

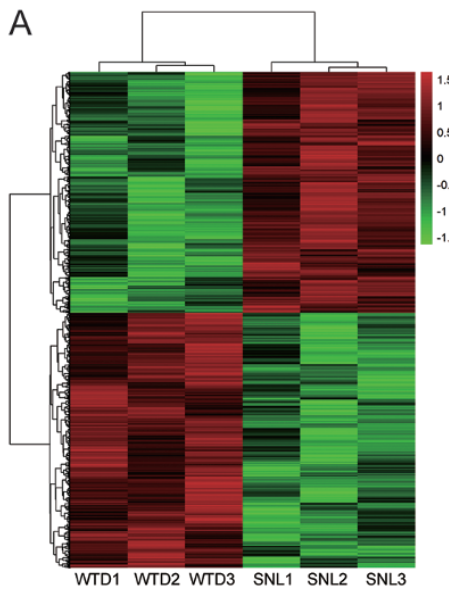

B

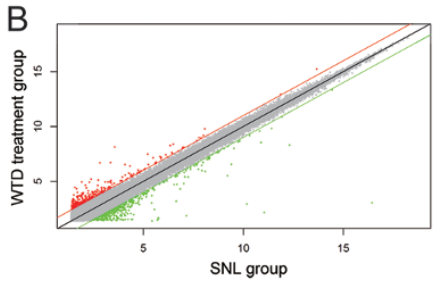

\section{Neuropathic pain state $\longrightarrow$ Pain recovery state}

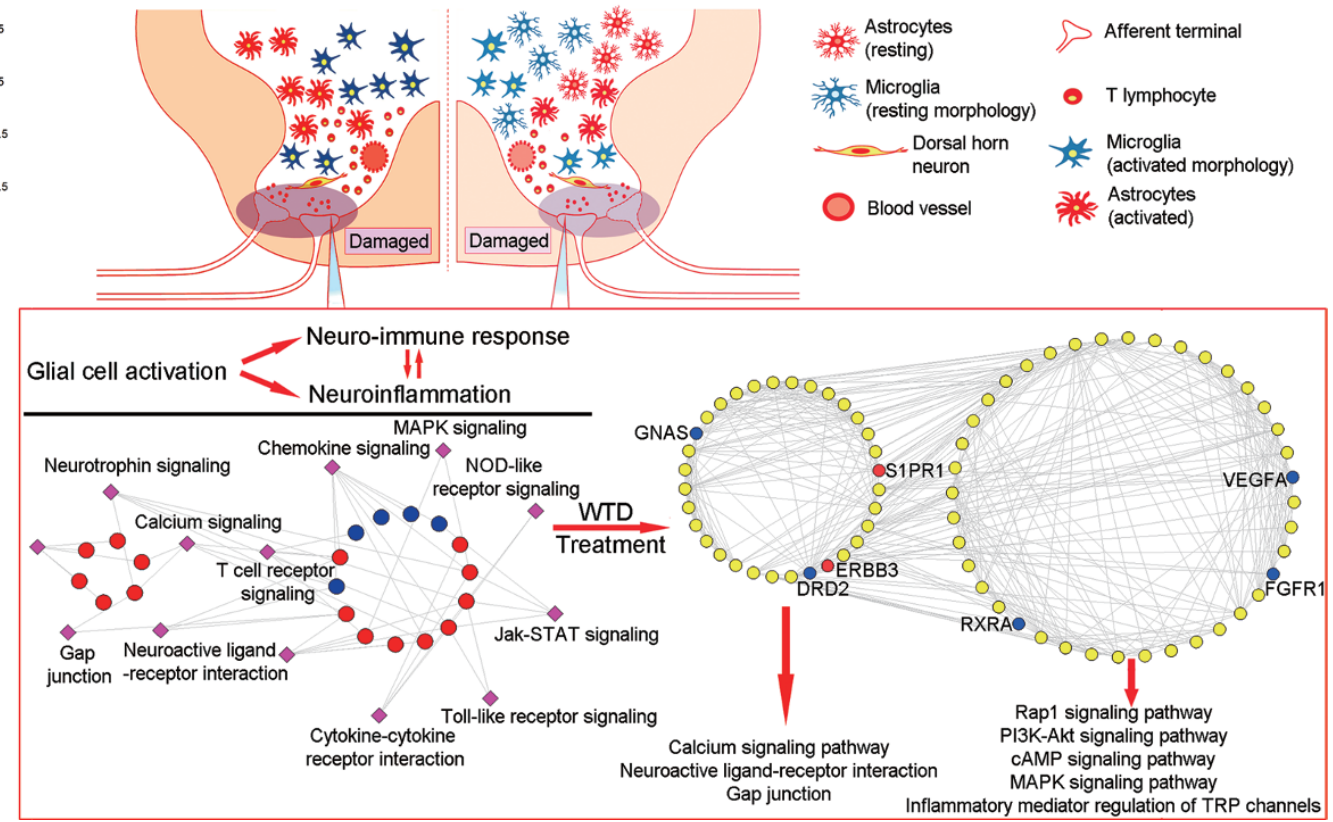

Figure 5. WTD exerts an analgesic effect on SNL-induced NP through the suppression of glial cell activation and neuroinflammation. (A) A heat map showing the hierarchical clustering of mRNAs, whose expression changes were increased by more than two-fold in the comparison between the SNL group and the WTD-treatment group. In the clustering analysis, up- and downregulated genes are colored in red and green, respectively. (B) A scatter plot comparing the global mRNA expression profile in the spinal cord between WTD-treated SNL mice and untreated SNL mice. Red and green lines, respectively, indicate two-fold differences in up- and downregulated genes. (C) The WTD-SNL-network constructed on the basis of the links among WTDtreatment dysregulated genes and SNL-induced NP-related genes. Red and blue nodes, respectively, refer to the upregulated and downregulated genes in the WTD treatment group compared with the SNL group. Yellow nodes refer to the known NP-related genes. 
ways that were involved in glial cell activation (Neuroactive ligand-receptor interaction, Calcium signaling pathway and Gap junction) and neuroinflammation (Rap1 signaling pathway, PI3K-Akt signaling pathway, cAMP signaling pathway, MAPK signaling pathway, Inflammatory mediator regulation of TRP channels and Cytokine-cytokine receptor interaction) (all $P<0.05$, Figure 5C). Seven WTD-treatment dysregulated genes, including DRD2, ERBB3, FGFR1, GNAS, RXRA, S1PR1 and VEGFA, were identified in the above NP-related pathways, thus suggesting that these genes may be candidate targets of WTD that act on SNL-induced NP.

\section{Discussion}

As common and intractable chronic pain, NP is caused by injuries or diseases that affect the nervous system ${ }^{[1]}$. The pathogenesis of NP is complicated and has not been fully elucidated. Growing evidence shows that the gene expression patterns of the nociceptive system may play crucial roles in the development and progression of $\mathrm{NP}^{[6-8]}$. The SNL model has been extensively used to investigate the underlying mechanisms of NP, in the current study, global expression changes in mRNAs of the spinal cord dorsal horn under the NP condition were identified on the basis of the SNL model, with rapid and persistent pain hypersensitivity characterized by mechanical hyperalgesia and heat hyperalgesia. Among the 41174 mRNAs detected in the spinal cord dorsal horn in the mice, $331 \mathrm{mRNAs}$ were upregulated and 236 mRNAs were downregulated at $10 \mathrm{~d}$ after SNL. In particular, 18 reported pain-related genes displayed differential expression patterns after SNL. According to the interactions among the SNLinduced DEGs and known NP-related genes, the SNL-induced NP imbalance network was constructed, and the hubs were screened. After pathway enrichment analysis of the hubs, we found that the significantly enriched pathways of the hubs in the SNL-induced NP imbalance network were mainly involved in glial cell activation, neuro-immune responses and neuroinflammation, which play crucial roles in the induction and maintenance of NP. Moreover, the levels of IL-1 $\beta$, TNFa, CCL2 and CXCL1 proteins in the spinal cord dorsal horn in SNL mice dramatically increased on the $10^{\text {th }} \mathrm{d}$ post-surgery, according to our ELISA analysis, thus suggesting activation of microglia and astrocytes. These data collectively indicated that suppression of glial cell activation, immune responses and inflammatory mediator production may be an effective way to attenuate NP.

Various therapeutic methods, such as non-pharmacological approaches (acupuncture and electric stimulation) and drugs (opioid analgesics, anti-convulsants, gabapentin, tricyclic antidepressants and local anesthetics), have been used to treat $\mathrm{NP}^{[5]}$. However, the treatment effectiveness has not been satisfactory, and side effects often occur. In recent years, several plant-derived analgesics have been indicated to be effective and non-toxic ${ }^{[25-28]}$. WTD, which contains five Chinese herbs, has previously been demonstrated to effectively treat joint diseases $^{[9]}$ and NP in clinics ${ }^{[11,12]}$ and inhibit chronic inflammatory pain in mice ${ }^{[10]}$. To determine whether it exerts an analgesic effect on NP, we examined the changes in mechanical hyperalgesia and heat hyperalgesia in the ipsilateral paw in SNL mice before or after WTD administration. Our data showed that orally administered intragastric WTD effectively attenuated mechanical hyperalgesia and heat hyperalgesia induced by SNL in time- and dose-dependent manners. An increasing number of studies indicate that activated microglia and astrocytes play important roles in the maintenance of NP. After their activation, glial cells may release inflammatory stimulants, such as cytokines, prostaglandins and neurotrophic factors, and may change the polarization features of the afferent neurons, thus leading to the modulation of pain signal transduction in the central nervous system ${ }^{[29,30]}$. Here, our data showed that WTD administration clearly suppressed the expression of microglia-releasing cytokines (IL$1 \beta$ and TNF- $\alpha$ ) and astrocyte-releasing cytokines (CCL2 and CXCL1) in the spinal cord dorsal horn in SNL mice in a dosedependent manner, thus suggesting an inhibitive role of WTD in the activation of these glial cells during the maintenance of NP. Together, these findings demonstrate the analgesic effect of WTD on NP.

To investigate the underlying pharmacological mechanisms of WTD acting on NP, we detected the gene expression profiles of the spinal cord dorsal horn obtained from SNL mice with or without WTD treatment. As a result of the DEG analysis, 442 out of 41174 studied transcripts exhibited changes in abundance after administration of WTD. Of these, 171 were upregulated, and 271 were downregulated. After the construction of the network of WTD-treatment dysregulated genes and SNL-induced NP-related genes as well as the topological calculation, 94 major nodes were identified. According to KEGG pathway enrichment analysis, these genes contributed to NP progression through a variety of glial cell activation-related pathways, including the Neuroactive ligand-receptor interaction, Calcium signaling pathway and Gap junction, as well as several neuroinflammatory pathways, including the cAMP signaling pathway, Rap1 signaling pathway, PI3K-Akt signaling pathway, MAPK signaling pathway, inflammatory mediator regulation of TRP channels and Cytokine-cytokine receptor interactions. cAMP signaling has been reported to play a critical role in the regulation of pain sensitivity. Singhmar et $a l^{[31]}$ have found that phosphorylation of cAMP1 at Ser-108 suppresses cAMP1-to-Rap1 signaling, thus leading to inhibition of persistent inflammatory pain. Three isoforms of AKT (AKT1-3) functionally regulate various cellular processes, such as transcriptional regulation, proliferation, apoptosis, and survival ability ${ }^{[32]}$. AKTs have been indicated to be downstream agents of PI3K signaling. PI3K-Akt signaling is known to mediate NP, and the inhibitor of this signaling may dosedependently attenuate pain behavior ${ }^{[33,34]}$. Phosphorylation of MAPKs and subsequent activation of MAPK signaling may regulate neuroinflammation, neuronal survival and synaptic activity $^{[35]}$. Many studies have revealed that MAPK signaling may play important roles in the mediation of the pain signaling cascade and may contribute to the maintenance of peripheral and central neuronal sensitization, as well as the activation 
of glial cells associated with $\mathrm{NP}^{[36-38]}$. TRP channels function as a specialized group of somatosensory ion channels that are involved in the detection of physical and chemical stimuli and in promotion of painful sensations via nociceptor activation ${ }^{[39]}$. Our previous study has demonstrated that the antinociceptive effect of WTD in chronic inflammatory pain may partly result from the inhibition of the activities of several TRPs, such as TRPV1, TRPA1, and TRPM8 ${ }^{[10]}$. Regarding NP, growing evidence indicates that targeting primary afferent nociceptive neurons that contain TRP channels may be an effective treatment modality for clinical pain syndromes ${ }^{[40]}$. As a proinflammatory cytokine-mediated process, neuroinflammation may be induced by systemic tissue injuries and may involve neuralimmune responses that activate immune cells, glial cells and neurons, thereby leading to $\mathrm{NP}^{[41]}$. Cytokine-cytokine receptor interactions may play an important role during this process. In recent years, several clinical trials with anti-cytokine agents, cytokine receptor antibodies and cytokine-signaling inhibitors have provided hope for future success in the treatment of NP. Because the WTD candidate targets were demonstrated to be involved in the above NP-related pathways, our findings may provide novel insights into the molecular basis of the analgesic effect of WTD in NP.

\section{Conclusions}

The current study clarified the gene expression patterns in the mouse spinal cord under the NP condition. The analgesic effects of WTD through suppression of glial cell activation and neuroinflammation were also demonstrated, thus suggesting the potential of WTD as a promising candidate for the treatment of NP. Further studies are required to determine the regulatory effects of WTD on the candidate targets in the treatment of SNL-induced NP.

\section{Acknowledgements}

This study was supported by the National Natural Science Foundation of China (No 81630107, 81573878, 81374068 and 81673834), and Beijing Nova program (№ Z1511000003150126).

\section{Author contribution}

Na LIN and Yan-qiong ZHANG conceived the study, participated in its design and coordination and helped draft the manuscript; Yan-qiong ZHANG and Chao WANG carried out the experiments and drafted the manuscript; Qiu-yan GUO, Chun-yan ZHU, Chen YAN, Dan-ni SUN, and Qiong-hong XU participated in the design of the study and performed the statistical analysis. All authors read and approved the final manuscript.

\section{Supplementary information}

Supplementary information is available at the website of the Acta Pharmacologica Sinica.

\section{References}

1 Colloca L, Ludman T, Bouhassira D, Baron R, Dickenson AH, Yarnitsky
D, et al. Neuropathic pain. Nat Rev Dis Primers 2017; 3: 17002.

2 Casale R, Symeonidou Z, Bartolo M. Topical treatments for localized neuropathic pain. Curr Pain Headache Rep 2017; 21: 15.

3 Thakur KK, Saini J, Mahajan K, Singh D, Jayswal DP, Mishra S, et al. Therapeutic implications of toll-like receptors in peripheral neuropathic pain. Pharmacol Res 2017; 115: 224-32.

4 Jones RC 3rd, Lawson E, Backonja M. Managing neuropathic pain. Med Clin North Am 2016; 100: 151-67.

5 Pessoa BL, Escudeiro G, Nascimento OJ. Emerging treatments for neuropathic pain. Curr Pain Headache Rep 2015; 19 : 56.

6 Rojewska E, Korostynski M, Przewlocki R, Przewlocka B, Mika J. Expression profiling of genes modulated by minocycline in a rat model of neuropathic pain. Mol Pain 2014; 10: 47.

7 Vallejo R, Tilley DM, Cedeño DL, Kelley CA, DeMaegd M, Benyamin R. Genomics of the effect of spinal cord stimulation on an animal model of neuropathic pain. Neuromodulation 2016; 19: 576-86.

8 Jiang BC, Sun WX, He LN, Cao DL, Zhang ZJ, Gao YJ. Identification of IncRNA expression profile in the spinal cord of mice following spinal nerve ligation-induced neuropathic pain. Mol Pain 2015; 11: 43.

9 Lv S, Wang Q, Li G, Sun S, Guo Y, Kuang H. The treatment of rheumatoid arthritis using Chinese medicinal plants: From pharmacology to potential molecular mechanisms. J Ethnopharmacol 2015; 176: 177-206.

10 Wang C, Liu C, Wan H, Wang D, Sun D, Xu T, et al. Wu-tou Decoction inhibits chronic inflammatory pain in mice: participation of TRPV1 and TRPA1 ion channels. Biomed Res Int 2015; 2015: 328707.

11 Liu GC. Therapeutic effects of Wu-tou Decoction on trigeminal neuralgia in clinics (observation of 24 cases). Acta Chin Med Pharmacol 1995; 6: 31.

12 Bai GH, Zhang JH. Therapeutic effects of Wu-tou Decoction on trigeminal neuralgia in clinics (observation of 24 cases). Modern J Integr Tradit Chin Western Med 2003; 12: 385.

13 Zhang Y, Wang D, Tan S, Xu H, Liu C, Lin N. A systems biology-based investigation into the pharmacological mechanisms of Wu Tou Tang acting on rheumatoid arthritis by integrating network analysis. Evid Based Complement Alternat Med 2013; 2013: 548498.

14 Zhang Y, Bai M, Zhang B, Liu C, Guo Q, Sun Y, et al. Uncovering pharmacological mechanisms of Wu-tou decoction acting on rheumatoid arthritis through systems approaches: drug-target prediction, network analysis and experimental validation. Sci Rep 2015; 5: 9463.

15 Zhang Y, Mao X, Guo Q, Bai M, Zhang B, Liu C, et al. Pathway of PPARgamma coactivators in thermogenesis: a pivotal traditional Chinese medicine-associated target for individualized treatment of rheumatoid arthritis. Oncotarget 2016; 7: 15885-900.

16 Zhang Y, Li Y, Mao X, Yan C, Guo X, Guo Q, et al. Thyroid hormone synthesis: a potential target of a Chinese herbal formula Haizao Yuhu Decoction acting on iodine-deficient goiter. Oncotarget 2016; 7: 51699-712.

17 Guo Q, Mao X, Zhang Y, Meng S, Xi Y, Ding Y, et al. Guizhi-ShaoyaoZhimu decoction attenuates rheumatoid arthritis partially by reversing inflammation-immune system imbalance. J Transl Med 2016; 14: 165.

18 Haiyu X, Yang S, Yanqiong Z, Qiang J, Defeng L, Yi Z, et al. Identification of key active constituents of Buchang Naoxintong capsules with therapeutic effects against ischemic stroke by using an integrative pharmacology-based approach. Mol Biosyst 2016; 12 : 233-45.

19 Guo Q, Zhong M, Xu H, Mao X, Zhang Y, Lin N. A systems biology perspective on the molecular mechanisms underlying the therapeutic effects of Buyang Huanwu Decoction on ischemic stroke. Rejuvenation Res 2015; 18: 313-25. 
20 Xu H, Zhang Y, Lei Y, Gao X, Zhai H, Lin N, et al. A systems biologybased approach to uncovering the molecular mechanisms underlying the effects of dragon's blood tablet in colitis, involving the integration of chemical analysis, ADME prediction, and network pharmacology. PLoS One 2014; 9: e101432.

21 Zhang Y, Guo X, Wang D, Li R, Li X, Xu Y, et al. A systems biologybased investigation into the therapeutic effects of Gansui Banxia Tang on reversing the imbalanced network of hepatocellular carcinoma. Sci Rep 2014; 4: 4154.

22 Kim SH, Chung JM. An experimental model for peripheral neuropathy produced by segmental spinal nerve ligation in the rat. Pain 1992; 50: 355-63.

23 Wishart DS, Knox C, Guo AC, Cheng D, Shrivastava S, Tzur D, et al. DrugBank: a knowledgebase for drugs, drug actions and drug targets. Nucleic Acids Res 2008; 36: D901-6.

24 Hamosh A, Scott AF, Amberger JS, Bocchini CA, McKusick VA. Online Mendelian Inheritance in Man (OMIM), a knowledgebase of human genes and genetic disorders. Nucleic Acids Res 2005; 33: D514-7.

25 Wu YE, Li YD, Luo YJ, Wang TX, Wang HJ, Chen SN, et al. Gelsemine alleviates both neuropathic pain and sleep disturbance in partial sciatic nerve ligation mice. Acta Pharmacol Sin 2015; 36: 1308-17.

26 Liu M, Zhou L, Chen Z, Hu C. Analgesic effect of iridoid glycosides from Paederia scandens (LOUR) MERRILL (Rubiaceae) on spared nerve injury rat model of neuropathic pain. Pharmacol Biochem Behav 2012; 102: 465-70.

27 Yang L, Li Y, Ren J, Zhu C, Fu J, Lin D, et al. Celastrol attenuates inflammatory and neuropathic pain mediated by cannabinoid receptor type 2. Int J Mol Sci 2014; 15: 13637-48.

28 Tang J, Li ZH, Ge SN, Wang W, Mei XP, Wang W, et al. The inhibition of spinal astrocytic JAK2-STAT3 pathway activation correlates with the analgesic effects of triptolide in the rat neuropathic pain model. Evid Based Complement Alternat Med 2012; 2012: 185167.

29 Tiwari V, Guan Y, Raja SN. Modulating the delicate glial-neuronal interactions in neuropathic pain: promises and potential caveats. Neurosci Biobehav Rev 2014; 45: 19-27.

30 Yamasaki R, Fujii T, Wang B, Masaki K, Kido MA, Yoshida M, et al. Allergic inflammation leads to neuropathic pain via glial cell activation. J Neurosci 2016; 36: 11929-45.
31 Singhmar P, Huo X, Eijkelkamp N, Berciano SR, Baameur F, Mei FC, et al. Critical role for Epac1 in inflammatory pain controlled by GRK2mediated phosphorylation of Epac1. Proc Natl Acad Sci U S A 2016; 113: $3036-41$.

32 Pillai VB, Sundaresan NR, Gupta MP. Regulation of Akt signaling by sirtuins: its implication in cardiac hypertrophy and aging. Circ Res 2014; 114: 368-78.

33 Sun R, Yan J, Willis WD. Activation of protein kinase B/Akt in the periphery contributes to pain behavior induced by capsaicin in rats. Neuroscience 2007; 144: 286-94.

34 Jiang SP, Zhang ZD, Kang LM, Wang QH, Zhang L, Chen HP. Celecoxib reverts oxaliplatin-induced neuropathic pain through inhibiting PI3K/ Akt2 pathway in the mouse dorsal root ganglion. Exp Neurol 2016; 275: 11-6.

35 Ramesh G. Novel therapeutic targets in neuroinflammation and neuropathic pain. Inflamm Cell Signal 2014; 1. pii: e111.

36 Wang JY, Chen SP, Gao YH, Qiao LN, Zhang JL, Liu JL. Effect of repeated electroacupuncture intervention on hippocampal ERK and p38MAPK signaling in neuropathic pain rats. Evid Based Complement Alternat Med 2015; 2015: 641286.

37 Galan-Arriero I, Avila-Martin G, Ferrer-Donato A, Gomez-Soriano J, Piazza S, Taylor J. Early treatment with UR13870, a novel inhibitor of p38 $\alpha$ mitogenous activated protein kinase, prevents hyperreflexia and anxiety behaviors, in the spared nerve injury model of neuropathic pain. Neurosci Lett 2015; 604: 69-74.

$38 \mathrm{Ni} \mathrm{HD}$, Yao M, Huang B, Xu LS, Zheng Y, Chu YX, et al. Glial activation in the periaqueductal gray promotes descending facilitation of neuropathic pain through the p38 MAPK signaling pathway. J Neurosci Res 2016; 94: 50-61.

39 Basso L, Altier C. Transient receptor potential channels in neuropathic pain. Curr Opin Pharmacol 2016; 32: 9-15.

40 Marwaha L, Bansal Y, Singh R, Saroj P, Bhandari R, Kuhad A. TRP channels: potential drug target for neuropathic pain. Inflammopharmacology 2016; 24: 305-17.

41 Myers RR, Campana WM, Shubayev VI. The role of neuroinflammation in neuropathic pain: mechanisms and therapeutic targets. Drug Discov Today 2006; 11: 8-20. 